\title{
Automated Navigation and Mobile Vehicle Control using Wireless Sensor Network Technology
}

\author{
Kar-Keung D. Young \\ Embedded Systems Institute and the \\ Department of Electronic and \\ Computer Engineering \\ The Hong Kong University of Science \\ and Technology \\ Clear Water Bay, Kowloon \\ Hong Kong SAR, People's Republic \\ of China \\ kkdyoung@ust.hk
}

\author{
Yong Quan Ou, Jun Tao Feng, Zhi \\ Liang Ou, Lun Hui Cai \\ Embedded Systems Facility \\ The Hong Kong University of Science \\ and Technology \\ Fok Ying Tung Research Institute, \\ Guangzhou \\ Guangdong, People's Republic of \\ China \\ mouseoogeust.hk \\ tomfeng@ust.hk \\ ozliang@ust.hk \\ esiallen@ust.hk
}

\author{
Ken Kin Man Cheng, Jason Kam On \\ Ho, and Timmy T M Tsang \\ Embedded Systems Institute \\ The Hong Kong University of Science \\ and Technology \\ Clear Water Bay, Kowloon \\ Hong Kong SAR, People's Republic \\ of China \\ kencheng@ust.hk \\ jasonho@ust.hk \\ emt immy@ust.hk
}

\begin{abstract}
This paper introduces a novel concept for automated navigation and control of a mobile platform. It utilizes an ad-hoc mobile wireless sensor network to provide navigational information to the mobile platform embedded control system. The embedded controller is realized with an ARM9 SOC based single board computer, with real time, multi-thread software implementation using a real time Linux operating system framwork. We have repeatedly tested the automated navigation and control concept on a wheel based mobile platform in an indoor environment, and found the performance meeting our original design goals.
\end{abstract}

\section{INTRODUCTION}

Automated guidance, navigation, and control of mobile platforms is key problem for any intelligent vehicle application. Often the enabling technologies are complex and implicitly costly. Computer vision has been applied to aid in the tracking and following of target human operator, in a master-servant context, and in a service robot application environment [1]. In this paper, we introduce an innovative approach to solve this critical problem for a class of mobile platforms which characteristically operates in an indoor environment. We devise a concept of target tracking which is based on ad-hoc network protocol and a wireless sensor network [2] with sensor nodes placed on-board the mobile platform as well as on the target. With this approach, identification and tracking of the target are accomplished concurrently, i.e., it is also possible to switch track on the fly if indeed there are multiple targets in the vincinity.

We first introduce the elctronic hardware aspects of the sensor node used in the system, followed by a description of the underlying software architecture for wireless sensor network applications. We outline the algorithmic aspects of target tracking, and illustrate the unique features of the wireless sensor network system which provide automatic node identification and other possible sensor driven intelligent control mechanisms. Finally, the interface between the target tracking subsystem and the mobile platform control system

\footnotetext{
*This work was supported by donation from the Fok Ying Tung Foundation to The Hong Kong University of Science and Technology
}

will be discussed. Real time system design aspects of the on-board embedded system will be summarized.

\section{WIRELESS SENSOR MODULE}

The wireless sensor module we have chosen for this application is based on Telos originally developed by the Computer Science Department at UC Berkeley. It belongs to a family of low power wireless sensor network devices have been built to enable research and deployments. The devices have featured commercial off the shelf (COTS) components integrated together on a platform commonly referred to as a "mote" [3]. Telos Rev B is a next-generation mote platform for ultra low power, high data-rate, wireless sensor network applications. It integrates an IEEE 802.15.4 compliant Chipcon Wireless Transceiver CC2420 [4], an on-board antenna providing up to 125 -meter range, and an ultra lower power Texas Instrument microcontroller TI MSP430 [5] into one circuit module. The $\mathrm{CC} 2420$ is a low power transceiver (17mA receive/19mA transmit) at 250kbps using O-QPSK. This module also offers a number of integrated peripherals including a 12-bit ADC and DAC, Timer, I2C, SPI, UART, and DMA controller, and an USB port for programming, debugging and data collection. The circuit's functional block diagram and the fabricated circuit PCB are shown in Fig. 1 and Fig. 2.

Software development for the wireless sensor module anchors on TinyOS [6], a small open source operating system for wireless network embedded systems, with an emphasis on reacting to external events and extremely low power operation. Rather than a monolithic OS, TinyOS is a set of components which are included as-needed in applications. TinyOs is implemented with the nesC language [7]. It is a component-based language with an event-based execution model. nesC components have similarities to objects: they encapsulate state and interact through well-defined interfaces. They also have significant differences: the set of components and their interactions are fixed at compile-time (to promote reliability and efficiency), rather than at run-time, as objectoriented references and instantiation do. 
At each node, transmit power is software programmable; transmitted range is subdivided into 32 discrete levels. The maximum transmitted range is up to 125 meters. Through testing, we determine that for range level 1 , the tranmitted range is within 0.5 meters, level 2 within 1.5 meters, and level 3 within 15 meters. Clearly, the relationship between the programmable levels and transmitted range is highly nonlinear; however, we have performed repeated experiments on a large number of sensor nodes, and arrived at the above observations.

\section{TARGET TRACKING ALGORITHM}

A wireless sensor network is built as the underlying hardware infrastructure on which the target tracking algorithm is conceptualized. Seven wireless sensor nodes with each node realized with a Telos Rev.B module form an ad-hoc mobile network. Six of the seven nodes are distributed on-board the mobile platform, with the seventh node - the Target node - colocated with the target to be tracked. In some sense, a cooperative target is subsumed in this design concept.

Fig. 3 is a photograph of the mobile platform with the wireless sensor nodes on-board. Among the six on-board nodes, five of then are the so-called direction nodes which are the main elements for detecting the target's directional information. Four of the direction nodes are physically mounted on the four edges of the mobile platform, i.e., Front, Back, Left and Right, with the corresponding node names, DirectionF, DirectionB, DirectionL, DirectionR. The fifth direction node is mounted at the center of the mobile platform - DirectionC. The sixth on-board node is called the Server node which is the node designated to communicate with the Target node. Fig. 4 illustrates the wireless sensor network layout and the directed communication paths among the network nodes. Fig. 5 shows the use case diagram of the wireless sensor network.

The on-board direction nodes are also referred to as satellite nodes since they are fixed relative to each other and periodically transmitting their respective ID tag information, similar to GPS. The integrated RF antenna is properly shielded at each of the satellite nodes. Their transmitted range are set to level 3 to reduce the necessary shielding. The directionality of the emitted RF signals allows the target node to identify the ID's of the satellite nodes that it can receive. We define a relative position vector $\vec{r}_{\text {target }} \equiv\left[r_{t x}, r_{t y}\right]$ from the center of the mobile platform to the target, and the associated relative angle $\alpha \equiv \arctan \frac{r_{t y}}{r_{t x}}$. With the node ID's cross reference with their relative on-board locations, the target node categorizes the relative position into eight directions as shown in Fig. 6 according to the decision logic table as shown in Fig. 7. In this table, the direction decoded if two or more sensor nodes are detected is shown. For example, if Target receives both DirectonF and DirectionL, the resulting direction is FrontLeft. If both DirectonF and DirectionB are received, there exists error, so an invalid message is generated. If three sensor nodes are detected, two of the three must be contradictory, thus the remaining sensor direction is decoded, . e.g. if DirectonF, DirectionB and DirectonR are detected, the direction decoded is Right. The decoded directions will be transmitted by Target and received by Server.

At the DirectionC node, the transmitted range is further reduce to level 2, and no shielding is applied. The primary role of this node is to detect proximity between the mobile platform and the target. At close range, Target will detect the presence of DirectionC which serves to provide critical information to enable collision avoidance functionality at the mobile platform control level. If the Target comes within 1.5 meters and being detected, it is considered to be too close a distance, and hence the mobile platform is commanded to stop motion. In this case, instead of transmitting a direction, Target will generate a "STOP" message to Server. In case of multiple sensor node detection which includes DirectionC, according to the decision logic table, a "STOP" message will be sent regardless.

A work flow chart of the implemented target tracking algorithm is given as Fig. 8. This diagram is in fact an activity diagram generated from the UML software engineering model.

\section{MOBILE PLATFORM CONTROL SYSTEM}

The mobile platform we have chosen is wheel-based. Two AC servo motors control repsectively a right and left wheel. The motion of this platform, it's velocity vector, is controlled by coordinating the speeds of the two powered wheels. Each of the two wheels is actuated with AC servo motor with worm gears. Closed loop velocity control of the wheel rotation is achieved through feedback of motor shaft mounted optical encoder signals. An interface circuit board with CPLD (Fig. 9) reads the encoder's A,B, and Z pulses and decodes the motor shaft's relative position to a data register which can be read from the on-board embedded computer system. An PID feedback control algorithm is implemented in a multi-thread, rate monotonic scheduled,real time software environment.

The direction decoded by the wireless sensor network can be expressed in terms of the relative angle $\alpha$. Using Fig. 10, we derive in this case the velocity differential between the left and right wheel as a function of $\alpha$ as follows:

$$
\begin{aligned}
V_{\text {right }} & =d \tan \alpha+V_{N} \\
V_{\text {left }} & =V_{N}
\end{aligned}
$$

where $V_{N}$ is the nominal velocity desired when the mobile platform moves along it's longitudinal axis, and $d$ denotes the distance between the right and left wheel. This is the case when the relative position vector $r_{\text {target }}$ lies in the Front-Left quadrant. For the case when $r_{\text {target }}$ lies in the Front-Right quadrant, $V_{\text {right }}=V_{N}$ and $V_{\text {left }}$ exceeds $V_{N}$ by the velocity differential component $d \tan \alpha$. Since tan function becomes infinitely large as $\alpha \rightarrow \pi / 2$, we implement a saturation value of $5 \times V_{N}$ on the velocity differential component. $V_{\text {right }}$ and $V_{\text {left }}$ will be used for wheel speed velocity set points for the right and left wheel motor speed control loops. For decoded directions in the Back-Right and Back-Left quadrants, we use the same equations to get the magnitudes of the velocity 
commands. The speed loop velocity set points will however be fixed so that the motors will drive the mobile platform in the Back directions.

\section{A. EMBEDDED COMPUTER SYSTEM}

Fig. 11 shows the single board computer (SBC) used for the development of the embedded mobile platform control system. This SBC builds around an $200 \mathrm{MHz}$ ARM9 920T core microporcessor with a maxuimum operating clock frquency of $200 \mathrm{MHz}$. A high performance Ethernet media access controlled is included along with external interfaces to SPI, AC'97 and $\mathrm{I}^{2} \mathrm{~S}$ audio; a two port USB 2.0 host, two UARTs and an analog-to-digital converter. The Telos Server node of the implemented wireless sensor network communicates with the SBC through one of the UART interface, as illustrated in Fig. 4.

\section{B. REAL TIME SOFTWARE ENGINEERING DEVELOP- MENT}

Real-Time Application Interface (RTAI) is a real-time Linux implementation. RTAI adds a small sub-kernel that runs under the standard Linux, and provides hard real-time response by running Linux as an idle task. RTAI provides a large selection of inter-process communication mechanisms and other real-time services. In the basic RTAI operation the real-time tasks are implemented as Linux kernel modules. RTAI intercepts the interrupts from peripherals and dispatches the interrupts to Linux kernel after handling the possible real-time actions triggered by the interrupts. Fig. 12 shows the system architecture of RTAI.

We adopt RTAI as the real time software implementation framework for the embedded control system for the mobile platform. Real time implementation of the sampled data feedback control loop is guaranteed by a rate monotonic periodic scheduler. The software engineering design is carried out using UML (Unified Modeling Language) 2.0 [8]. Fig. 13 shows the sequence diagram of the motion control thread execution. As shown in this diagram, the threads are delegated by the :scheduler in which a scheduler table defines the execution time and sequence of the different threads. At the beginning of sampling period, the :motorController issues a control command to drive the servo motor, and as well as reads the shaft speed data. PID algorithm calculation which is peformed by : calculator and UART communication with the Server node of the wireless sensor network which is performed by :communication are completed before the end of the period.

\section{EXPERIMENTAL RESULTS}

The mobile platform has been tested for a long period of time in an indoor environment. The proposed target tracking algorithm performed well as indicated by the correctness of the platform's turning performance as the target making a right hand or left hand turn respectibe to the platform. Emergency stop has also been tested to be functional: as the target moved toward the moving platform, the platform comes to a complete stop and beyond the designated 1.5 meters off limit perimeter. Fig. 14 shows the sequentially captured frames of a test run with the mobile platform following the target - one of our developers with the Target node in his hand.

\section{CONCLUSIONS}

In this paper, we have demonstrated a novel utilization of wireless sensor network in automated mobile platform navigation. The ad-hoc nature of the network provides many interesting extensions of the present work; one of which is the automated navigation of a pool of mobile platforms. Since the wireless sensor node has an embedded OS, additional network communication protocols can be developed to perform auto-registration and navigational decision making for any mobile platforms enabled with a compatiable sensor node sharing the same software and hardware architecture.

\section{ACKNOWLEDGMENTS}

We wish to thank Professor Lionel Ni of the Department of Computer Science and Engineering at the Hong Kong University of Science and Technology for his encouragement and support on our wireless sensor module and network development.

\section{REFERENCES}

[1] Y. S. Moon, K. H. Pun, K. C. Chan, M. F. Wong, T. H. Yu, ”Enabling EBGM Face Authentication on Mobile Devices", Proceedings of the Second International Workshop on MultiModal User Authentication, Toulouse, France, May 2006

[2] G. Aggelou, Mobile Ad Hoc Networks, McGraw Hill, New York, NY; 2005.

[3] Jason Hill, System Architecture for Wireless Sensor Networks. PhD Thesis, University of California, Berkeley, 2003.

[4] Chipcon AS CC2420 2.4GHz IEEE 802.15.4 compliant RF Transceiver. November 2003. http://www.chipcon.com

[5] Texas Instruments MSP430 Microcontroller: F1611 User's Guide, 2004. http://www.ti.com.

[6] David Gay, Philip Levis, and David Culler, "Software Design Patterns for TinyOS", Proceedings of the ACM SIGPLAN/SIGBED 2005 Conference on Languages, Compilers, and Tools for Embedded Systems (LCTES'05), Chicago, June 2005. http://www.tinyos.net.

[7] D. Gay, P. Levis, R. von Behren, M. Welsh, E. Brewer, and D. Culler, The nesC language: A holistic approach to networked embedded systems, SIGPLAN Conference on Programming Language Design and Implementation (PLDI03), June 2003.

[8] Bruce P. Douglass, Real time UML: advances in the UML for real-time systems, Third Edition, Addison Wesley, 2004. 


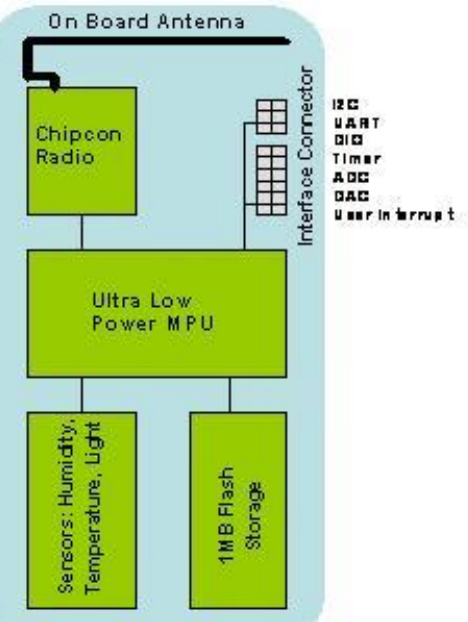

Fig. 1. Telos block diagram

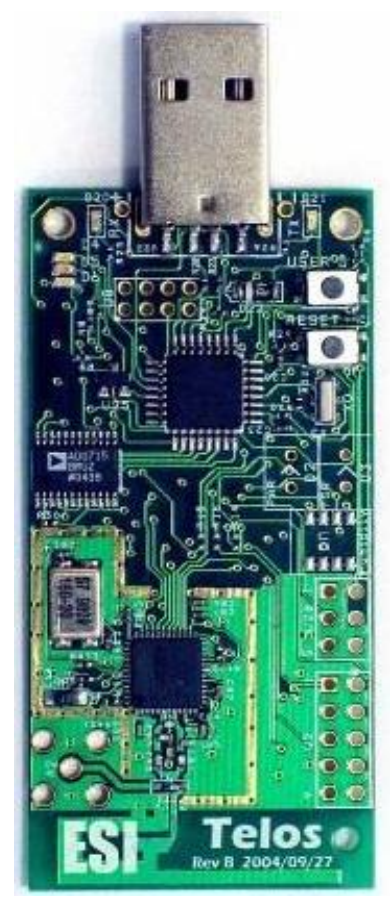

Fig. 2. Telos Rev. B PCB 


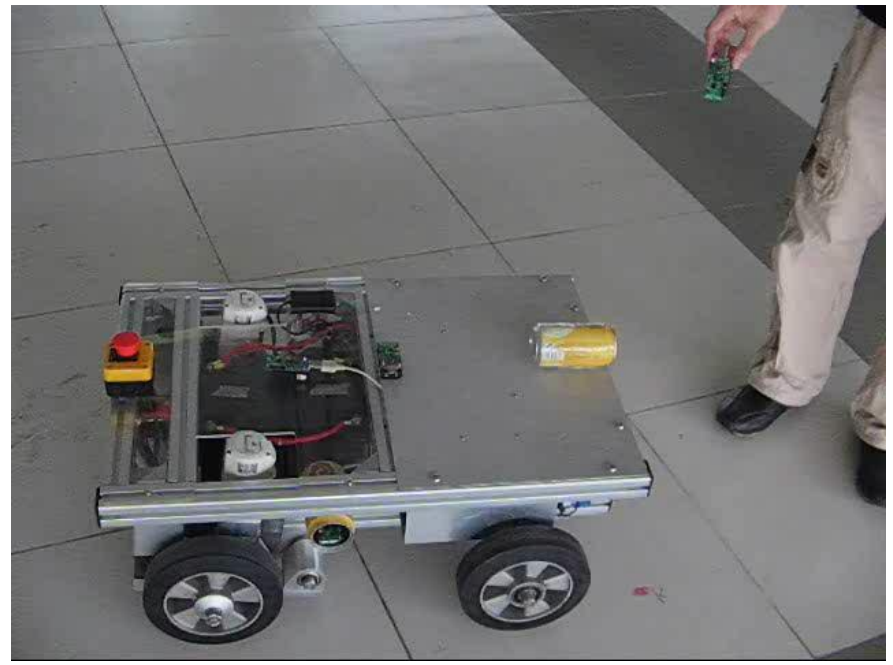

Fig. 3. Mobile Platform with wireless sensor nodes on board

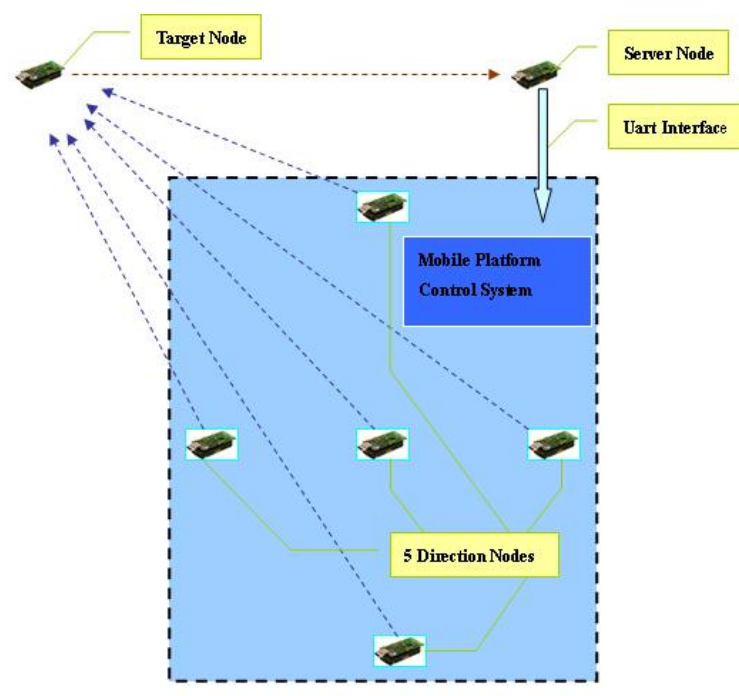

Fig. 4. Wireless sensor network and communication paths

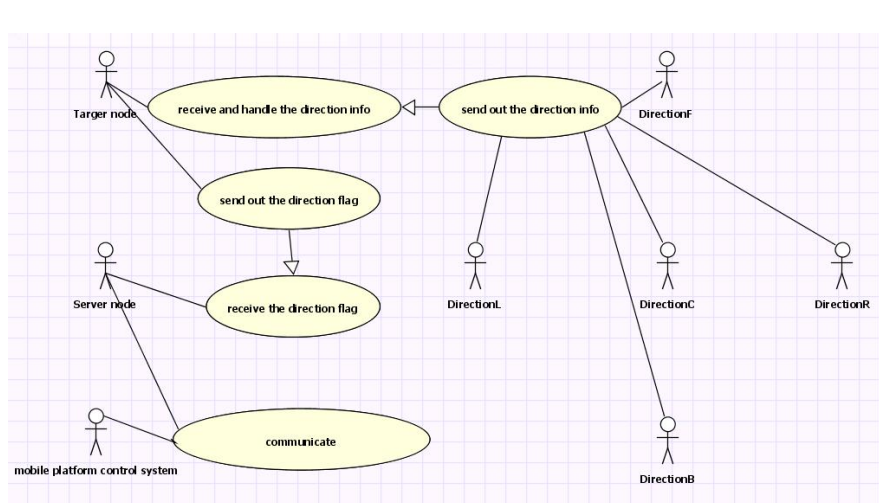

\begin{tabular}{|c|c|c|c|c|c|}
\hline Satellite Sensor Node & DirectionF & Dinection B & Direction L & Direction $\mathrm{R}$ & DirectionC \\
\hline Direction $F$ & Front & & & & \\
\hline Direction B & Error: Invalid & Back & & & \\
\hline Direction I & FrontLeft & BackLeft & Left & & \\
\hline Direction $\mathrm{R}$ & FrontRight & BackRight & Error: Invalid & 敒敒 & \\
\hline DirectionC & STOP & STOP & STOP & STOP & STOF \\
\hline
\end{tabular}

Fig. 7. Decision logic table

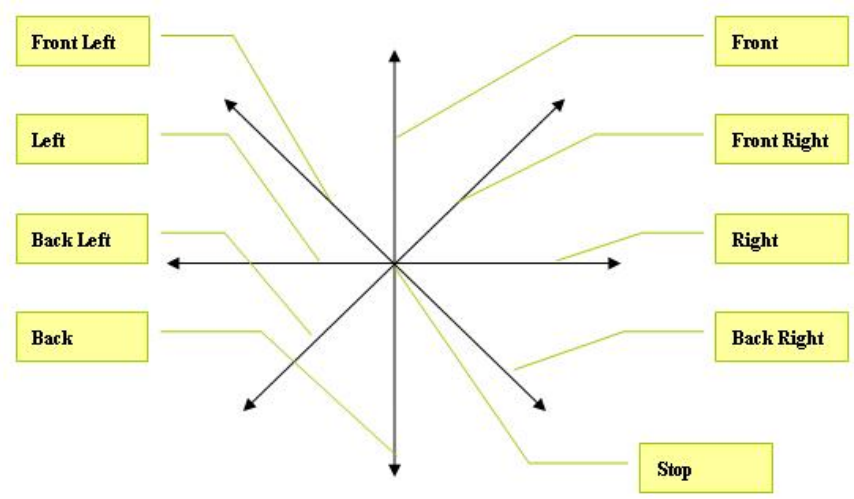

Fig. 6. Eight possible directions

Fig. 5. UML use case diagram of wireless sensor network 


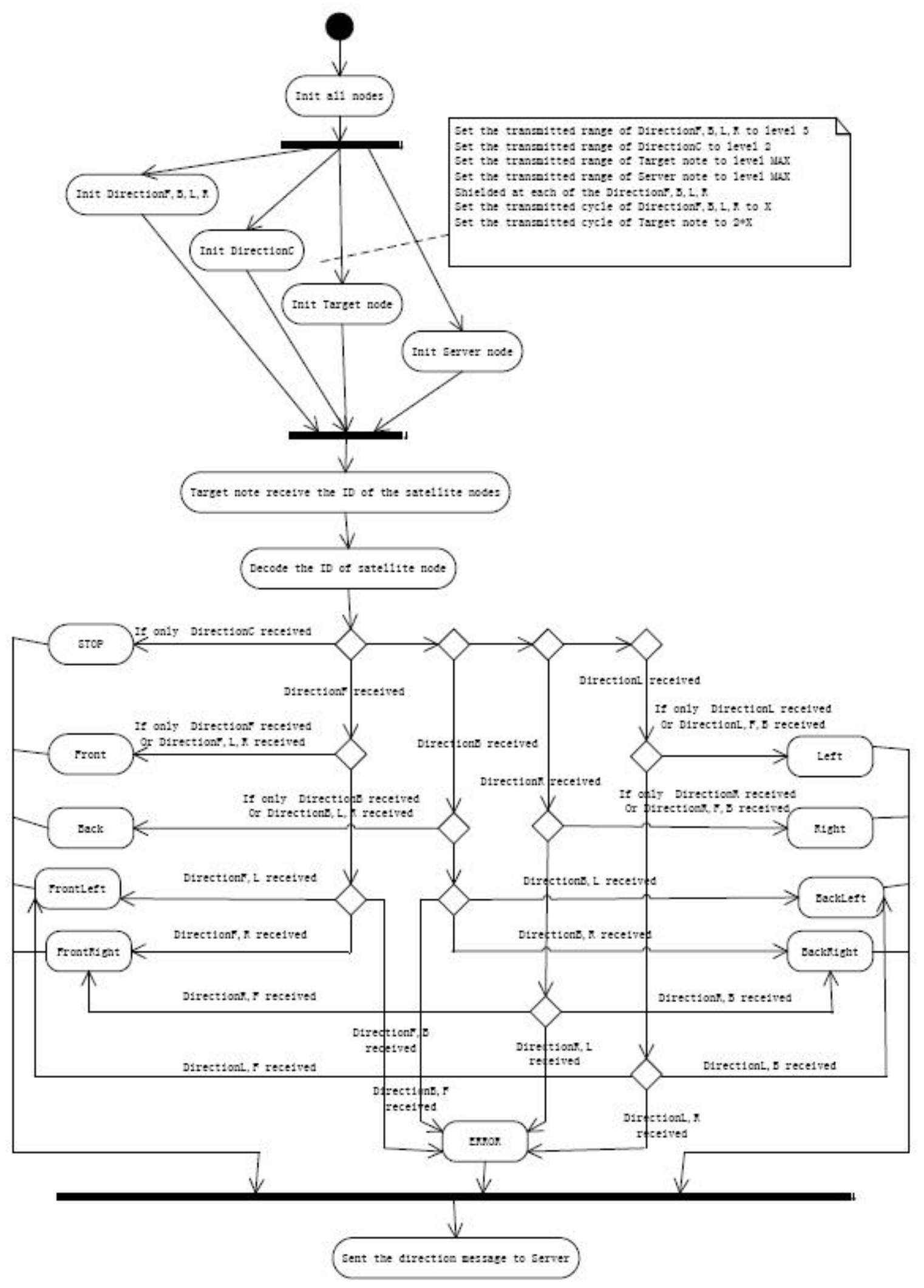

Fig. 8. UML Activity Diagram showing the target tracking work flow 


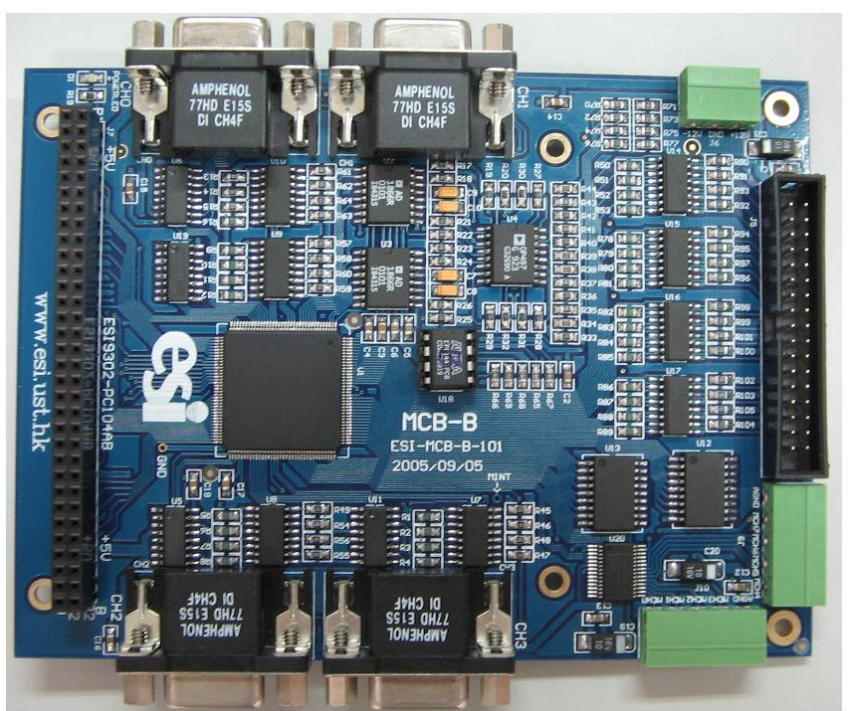

Fig. 9. Motor interface board

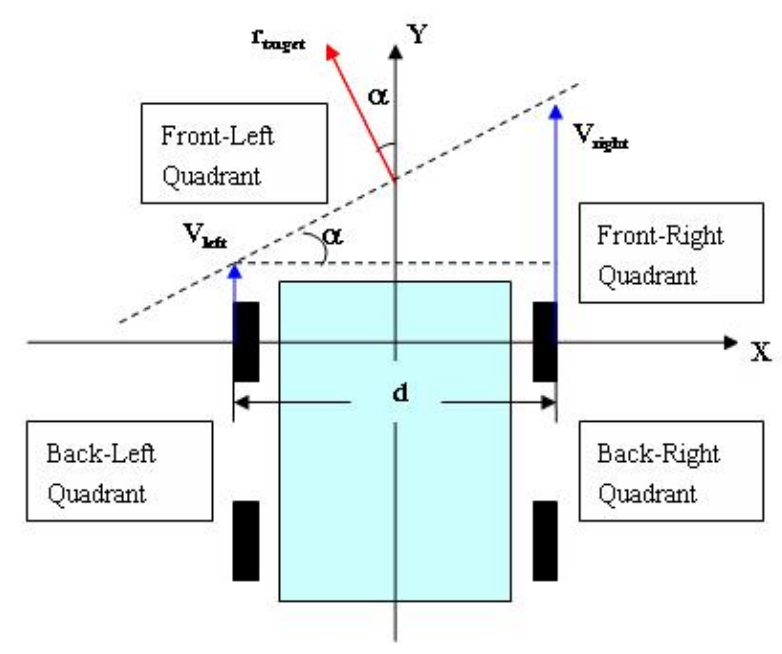

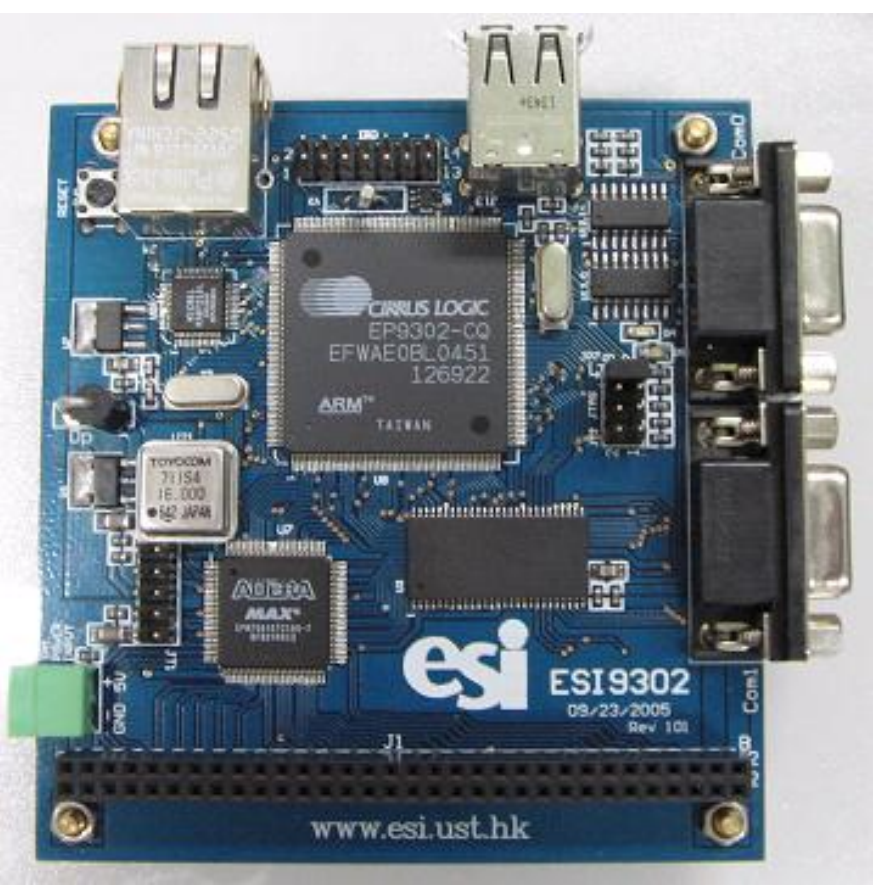

Fig. 11. ARM 9 based single board computer

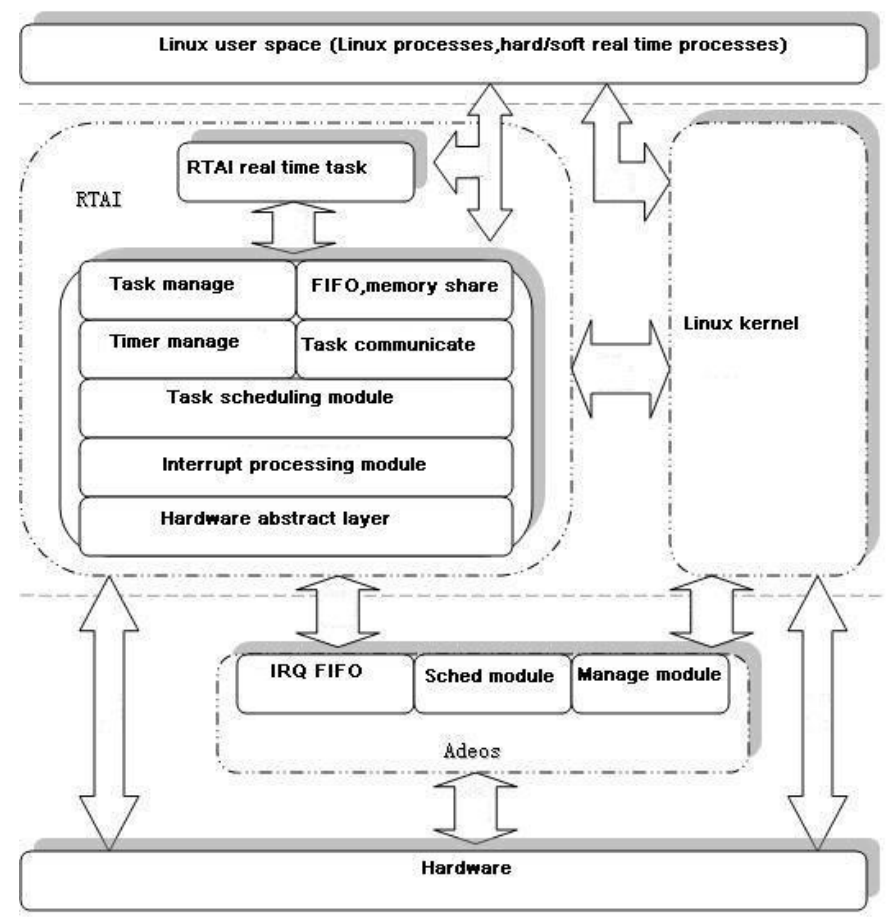

Fig. 10. Relative postion geometry between target and mobile platfrom

Fig. 12. RTAI Architecture 


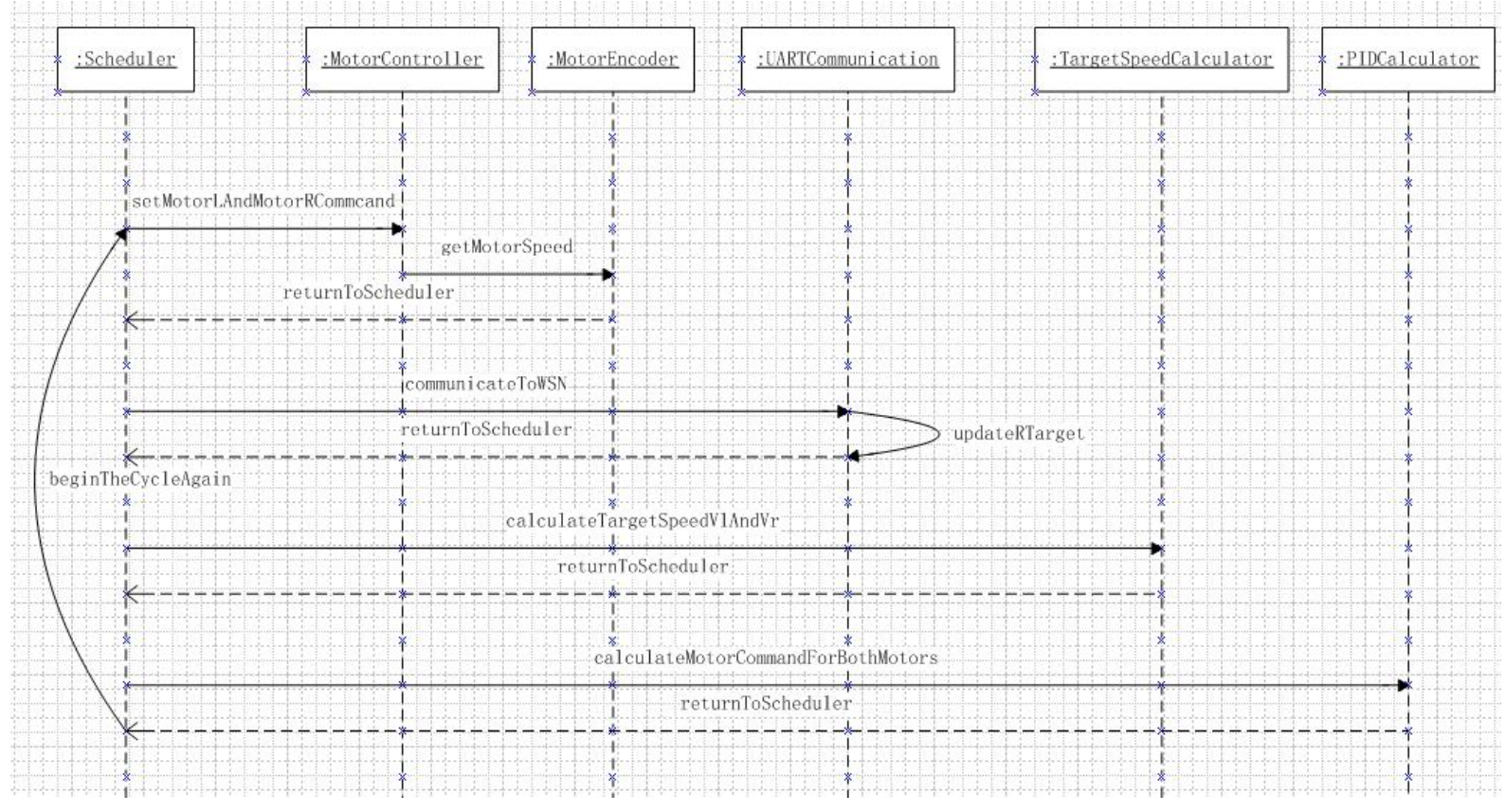

Fig. 13. Mobile platform control sequence diagram

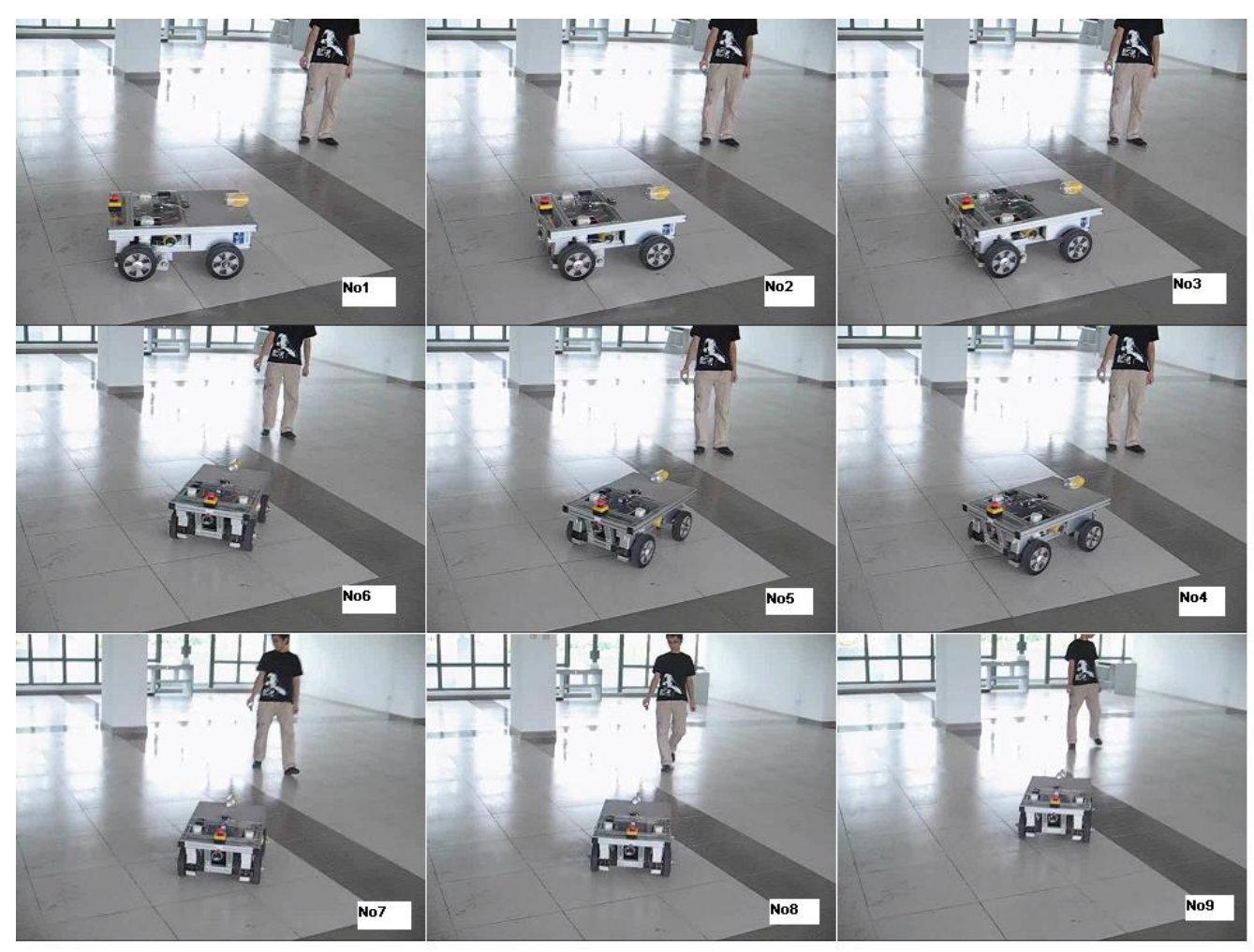

Fig. 14. Sequential captured frame of a test run 